\title{
FINITE COVERINGS BY COSETS OF NORMAL SUBGROUPS
}

\author{
M. M. PARMENTER
}

(Communicated by Warren J. Wong)

\begin{abstract}
In this brief note, we characterize those groups $G$ which can be covered by finitely many cosets $a_{i} M_{i}$ of maximal normal subgroups $M_{i}$, where the covering is irredundant and not all $M_{i}$ are equal. This refines an earlier result of Brodie, Chamberlain, and Kappe, who characterized those groups which can be covered by finitely many proper normal subgroups.
\end{abstract}

A group $G$ is said to be covered by a collection of cosets of subgroups if each element of the group belongs to at least one of the cosets. The covering is said to be irredundant if each of the cosets contains at least one element which belongs to no other coset.

The case where each coset in a covering is actually a normal subgroup has been investigated in a recent paper by Brodie, Chamberlain, and Kappe [3]. One of their main results is the following theorem.

Theorem 1. A group can be covered by finitely many proper normal subgroups if and only if it has a quotient isomorphic to an elementary abelian p-group of rank two for some prime $p$.

B. H. Neumann [6] had previously shown that a group can be covered by finitely many proper subgroups (not necessarily normal) if and only if the group has a finite noncyclic quotient.

Various questions involving coverings by cosets which are not necessarily subgroups have been studied by several authors-Berger, Felzenbaum, and Fraenkel [1], or Parmenter [7], for example. Much of the impetus for these papers comes from number theory, where a great deal of work has been done on problems concerning covering the integers by sets of arithmetic progressions (see [2] or [8]).

In this paper, we seek to extend Theorem 1, as stated above, to coverings by cosets of normal subgroups. As a by-product of this investigation, we obtain a different (perhaps simpler) proof of Theorem 1 than that given in [3].

Received by the editors September 13, 1989 and, in revised form, March 5, 1990.

1980 Mathematics Subject Classification (1985 Revision). Primary 20 F99.

This research was supported in part by NSERC grant A-8775. 
Since a group can always be covered by the complete set of cosets of any subgroup, some condition is needed to avoid this situation. Unfortunately, these trivial coverings are not always immediately recognizable, as can be seen in the following example given by Herzog and Schönheim [4]. Let $G=\left\langle x_{1}, x_{2}, x_{3}, x_{4}\right\rangle$ be the elementary abelian group of order 16 . Observe that $G$ is the union of the 5 cosets $\left\langle x_{2}, x_{3}, x_{4}\right\rangle,\left\langle x_{2}\right\rangle+x_{1}+x_{4},\left\langle x_{3}\right\rangle+x_{1}+x_{2},\left\langle x_{4}\right\rangle+x_{1}+x_{3}$, $\left\langle x_{2}+x_{3}+x_{4}\right\rangle+x_{1}$, and that all the subgroups are different. However, the union of the last four cosets is just $\left\langle x_{2}, x_{3}, x_{4}\right\rangle+x_{1}$, so we really have a trivial covering here, but in disguised form.

Note, however, that a group $G$ has a finite covering by subgroups if and only if it has a finite covering by maximal subgroups, and if our attention is restricted to the latter setting, the following result is obtained.

Theorem 2. For a group $G$, the following are equivalent:

(i) $G$ is covered by a finite set of cosets $a_{i} M_{i}$, with $M_{i}$ a maximal normal subgroup of $G$ for all $i$, such that the covering is irredundant and not all $M_{i}$ are equal.

(ii) $G$ has a quotient isomorphic to an elementary abelian p-group of rank two for some prime $p$.

As in all work of this type, the following result, due to B. H. Neumann, is crucial.

Lemma [5]. Let $G$ be covered by a finite set of cosets $a_{i} G_{i}$. If we omit from this covering any coset $a_{i} G_{i}$ for which $\left[G: G_{i}\right]$ is infinite, then $G$ is still covered by the remaining cosets.

We now proceed to prove our result.

Proof of Theorem 2. To prove (ii) $\Rightarrow$ (i), note that (ii) easily implies that $G$ can be covered by finitely many proper normal subgroups, and as remarked earlier this leads to (i).

Assume (i). By the lemma, and the fact that the covering is irredundant, we know that $\left[G: M_{i}\right]<\infty$ for all $i$. Passing to the finite quotient $G / M$, where $M=\bigcap M_{i}$, we may assume $M=1$.

Now select any $j$ and let $S=\left\{i \mid M_{i} \neq M_{j}\right\}$. Because of our assumption that not all $M_{i}$ are equal, $S$ is nonempty.

We know already that $\bigcap M_{i}=1$, and we will now show that $\bigcap_{i \in S} M_{i}=1$. Suppose this is not true and let $B=\bigcap_{i \in S} M_{i} \neq 1$. Because $M_{j}$ is a maximal normal subgroup of $G$ and $B \cap M_{j}=1$, we know that $G=B \times M_{j}$. It follows that, for each coset of $M_{j}$ present in the covering of $G$, we can assume that the coset representative is in $B$. We may also assume, by multiplying all cosets in the covering by a group element if necessary, that $M_{j}$, the coset of 1 , is part of our covering. Finally, note that some $d M_{j}$, where $d \neq 1$ is in $B$, is not part of the covering. 
Since the covering is irredundant, there exists $m$ in $M_{j}$ which does not belong to any other coset in the covering. But it is then easy to see that $d m$ cannot belong to any of the cosets in the covering (note that, by the above, $d$ is contained in every $M_{i} \neq M_{j}$, and $d m=m d$ since $B \cap M_{j}=1$ ), and this gives our contradiction. We conclude that $B=1$.

Next, choose $L$, a set of minimal cardinality such that $\bigcap_{i \in L} M_{i}=1$. Note that if $i \neq j$, with $i$ and $j$ in $L$, then $M_{i}$ and $M_{j}$ are distinct. From the previous work, we know that there exists $M_{\ell}$ such that $M_{\ell} \neq M_{i}$ for all $i$ in $L$.

Let $j$ be any member of $L$, and let $A$ be the intersection of the sets $M_{i}$, where $i$ is in $L$ but $i \neq j$. By minimality, we know that $A \neq 1$ and $A \cap M_{j}=$ 1 . Since $G / M_{j}$ is simple, $G=A M_{j}$, and this means that $A \simeq G / M_{j}$ is simple. Now $M_{\ell} \neq M_{j}$ allows us to choose $z=a b$ in $M_{\ell}$, with $a$ in $A, a \neq 1$, and $b$ in $M_{j}$.

We claim that the simple group $A$ must be abelian. Assume not; then there exists $c$ in $A$ such that $c a c^{-1} \neq a$. Now $M_{\ell} \triangleleft G$ implies $c z c^{-1}$ is in $M_{\ell}$, and $c z c^{-1}=c a c^{-1} c b c^{-1}=c a c^{-1} b$ since $A \cap M_{j}=1$, so $c z c^{-1} z^{-1}=c a c^{-1} a^{-1} \neq$ 1. It follows that $M_{\ell} \cap A \neq 1$. Since $A$ is simple, this means that $A \subseteq M_{\ell}$. Because this is true for all $M_{\ell}$ of the type described, we conclude that $A$ must be equal to the subgroup $B$ defined earlier. Since we saw that this latter subgroup is trivial, there is a contradiction. Thus $A$ must be abelian, i.e., $G / M_{j}$ is cyclic of prime order.

Since the above argument works for all $j$ in $L$ and $\bigcap_{j \in L} M_{j}=1$, we conclude that $G$ is abelian. If the primes $\left[G: M_{j}\right.$ ] were all distinct, $G$ would by cyclic of order $n=p_{1} p_{2} \cdots p_{t}$ (where the $p_{i}$ are the distinct primes). But in this case, a coset of a maximal subgroup is just a congruence modulo $p_{i}$ for some $i$. A covering of the type described in (i) must omit at least one congruence $x \equiv a_{i}\left(\bmod p_{i}\right)$ for each $i$, and the Chinese Remainder Theorem then tells us that some element of $G$ is not covered.

Hence, two of the primes $\left[G: M_{j}\right]$ must be equal, and we have the result.

Remarks. 1. If $G$ is covered by finitely many proper normal subgroups, then $G$ must satisfy condition (i), as remarked earlier. Hence Theorem 1 can be derived as a corollary of Theorem 2, giving an alternative approach to the proof of Theorem 1 from that given in [3]. In any case, Theorems 1 and 2 together tell us that any group which satisfies condition (i) can also be covered by finitely many proper normal subgroups.

2. Theorem 2 is clearly not true if the word "maximal" is omitted. To see this in a trivial fashion, let $G$ be any finite group with proper normal subgroups $M, N$ where $N$ is strictly contained in $M$-first express $G$ as a union of all cosets of $M$, and then decompose one of these cosets into a union of cosets of $N$.

3. The author would like to thank the referee for making several very helpful suggestions. 


\section{REFERENCES}

1. Marc A. Berger, Alexander Felzenbaum, and Aviezri Fraenkel, The Herzog-Schönheim Conjecture for finite nilpotent groups, Canad. Math. Bull. 29 (1986), 329-333.

2. __, New results for covering systems of residue sets, Bull. Amer. Math. Soc. 14 (1986), 121-125.

3. M. A. Brodie, R. F. Chamberlain, and L.-C. Kappe, Finite coverings by normal subgroups, Proc. Amer. Math. Soc. 104 (1988), 669-674.

4. M. Herzog and J. Schönheim, Research problem no. 9, Canad. Math. Bull. 17 (1974), 150.

5. B. H. Neumann, Groups covered by permutable subsets, J. London Math. Soc. 29 (1954), 236-248.

6. __ Groups covered by finitely many cosets, Publ. Math. Debrecen 3 (1954), 227-242.

7. M. M. Parmenter, Exact covering systems for groups, Fund. Math. 123 (1984), 133-136.

8. S. Znám, A survey of covering systems of congruences, Acta Math. Univ. Comenian. 40-41 (1982), 59-78.

Memorial University of Newfoundland, St. John's, Newfoundland A 1C 5S7 Canada 